\title{
Contrast-Enhanced PET/MR Imaging Versus Contrast- Enhanced PET/CT in Head and Neck Cancer: How Much MR Information Is Needed?
}

\author{
Felix P. Kuhn ${ }^{1}$, Martin Hüllner ${ }^{1}$, Caecilia E. Mader ${ }^{1}$, Nikos Kastrinidis ${ }^{2}$, Gerhard F. Huber ${ }^{2}$, Gustav K. von Schulthess ${ }^{1}$, \\ Spyros Kollias ${ }^{1}$, and Patrick Veit-Haibach ${ }^{1}$ \\ ${ }^{1}$ Department of Medical Radiology, University Hospital Zurich, Zurich, Switzerland; and ${ }^{2}$ Department of Otorhinolaryngology, \\ University Hospital Zurich, Zurich, Switzerland
}

Considering PET/MR imaging as a whole-body staging tool, scan time restrictions in a single body area are mandatory for the costeffective clinical operation of an integrated multimodality scanner setting. It has to be considered that ${ }^{18} \mathrm{~F}-\mathrm{FDG}$ already acts as a contrast agent and that under certain circumstances MR contrast may not yield additional clinically relevant information. The concept of the present study was to understand which portions of the imaging information enhance the sensitivity and specificity of the hybrid examination and which portions are redundant. Methods: One hundred fifty consecutive patients referred for primary staging or restaging of head and neck cancer underwent sequential wholebody ${ }^{18} \mathrm{~F}-\mathrm{FDG}$ PET with CT-based attenuation correction, contrast-enhanced (ce) CT, and conventional diagnostic MR imaging of the head and neck in a trimodality PET/CT-MR system. Assessed were image quality, lesion conspicuity, diagnostic confidence, and the benefit of additional coronal and sagittal imaging planes in cePET/CT, PET/MR imaging with only T2-weighted fat-suppressed images (T2w PET/MR imaging), and cePET/MR imaging. Results: In 85 patients with at least 1 PET-positive lesion, 162 lesions were evaluated. Similar robustness was found for CT and MR image quality. T2w PET/MR imaging performed similarly to (metastatic lymph nodes) or better than (primary tumors) cePET/CT in the morphologic characterization of PET-positive lesions and permitted the diagnosis of necrotic or cystic lymph node metastasis without application of intravenous contrast medium. CePET/MR imaging yielded a higher diagnostic confidence for accurate lesion conspicuity (especially in the nasopharynx and in the larynx), infiltration of adjacent structures, and perineural spread. Conclusion: The results of the present study provide evidence that PET/MR imaging can serve as a legitimate alternative to PET/CT in the clinical workup of patients with head and neck cancers. Intravenous MR contrast medium may be applied only if the exact tumor extent or infiltration of crucial structures is of concern (i.e., preoperatively) or if perineural spread is anticipated. In early assessment of the response to therapy, in follow-up examinations, or in a whole-body protocol for non-head and neck tumors, T2w PET/MR imaging may be sufficient for coverage of the head and neck. The additional MR scanning time may instead be used for advanced MR techniques to increase the specificity of the hybrid imaging examination.

Received May 1, 2013; revision accepted Sep. 30, 2013.

For correspondence or reprints contact: Felix P. Kuhn, Department of Medical Radiology, University Hospital Zurich, CH-8091 Zurich, Switzerland. E-mail: Felix.Kuhn@usz.ch

Published online Feb. 3, 2014.

COPYRIGHT (C 2014 by the Society of Nuclear Medicine and Molecular Imaging, Inc.
Key Words: PET/MRI; PET/CT; oncological imaging; head and neck cancer

J Nucl Med 2014; 55:551-558

DOI: 10.2967/jnumed.113.125443

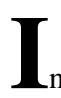
ntegrated PET/CT has been established as an important diagnostic technique for staging and therapy assessment in advanced head and neck cancers. ${ }^{18}$ F-FDG PET/CT has a higher sensitivity than CT or MR imaging for the detection of small lymph nodes (1). Also, in therapy assessment PET/CT has been shown to differentiate early responders from nonresponders (2). However, ${ }^{18} \mathrm{~F}$ FDG is known not to be specific to cancer cells, and inflammatory or physiologic uptake has to be considered as a differential diagnosis. Furthermore, cystic lymph node alterations may be overlooked. Therefore, contrast-enhanced (ce) PET/CT is advisable for the correct diagnosis of necrotic or cystic lymph node metastases (3). In addition, anatomic localization of ${ }^{18}$ F-FDG-positive lesions, determination of exact tumor extent, and determination of infiltration of adjacent structures such as muscles, fasciae, vessels, and cranial nerves is not reliably achieved with PET alone. Hence, the integration of functional and anatomic information in PET/CT significantly improves lesion localization and characterization (4).

Recently, several research groups have started to study the potential benefits of replacing CT by MR imaging in PET/MR imaging systems. For single-modality CT and MR imaging, there is continued controversy in the literature about whether ceCT or ceMR imaging is the superior modality for imaging head and neck tumors $(5,6)$. CT is a fast and robust imaging technique and offers excellent details on bony structures but shows important metallic and dental artifacts, has low soft-tissue contrast, and exposes the patient to ionizing radiation. MR imaging provides a substantially higher soft-tissue contrast and offers a wide range of additional imaging techniques $(7,8)$ such as apparent diffusion coefficient maps for the quantification of cellular density (9), arterial spin labeling, intravoxel incoherent motion, and dynamic contrast enhancement to assess the characteristics of tumor vasculature $(10,11)$. This additional information may help to increase the specificity in the characterization of PET-positive lesions.

However, MR imaging using advanced imaging techniques and multiple pulse sequences is time consuming and needs to be limited to a body region of particular interest-for example, the neck-in the framework of an integrated PET/MR examination. In 
combination with PET and as a whole-body staging tool, restriction of MR scanning time in a single body area is mandatory for the cost-effective clinical operation of an integrated multimodality scanner setting (12). A partial-body PET scan from the thigh to the vertex of the skull is done in 2- to 4-min scanning time slots per bed position. During such a step, MR imaging in the corresponding region has to be performed to provide morphologic images for anatomic localization, characterization of the PET findings, and possibly attenuation correction. In an integrated multimodality system, because of advantages in patient workflow, an additional 10 to, maximally, 15 min of dedicated MR imaging can be performed (12). This limitation greatly restricts the number of MR pulse sequences that can be applied. However, it has been recognized in early PET/CT research that a low-dose CT scan $(120 \mathrm{kV}$, $40 \mathrm{mAs}$ ) is, in some clinical questions, sufficient to serve as a morphologic correlate to the functional PET information (4), because some information obtained from one modality in a hybrid system is redundant with that obtained from the other modality. Hence, in integrated-modality protocols it is not necessary to optimize the sensitivity and specificity of each separate examination but rather of the combined examination. To give an example: a PET-positive lymph node frequently may not also need characterization by CT or MR imaging in a hybrid system; CT or MR imaging has only to demonstrate that the activity focus is there and that it does not superimpose with fatty tissue. Clinical studies with PET/MR will show when the restricted use of MR pulse sequences is adequate and when additional data have to be acquired in the major body regions of interest.

The purpose of this prospective study was to assess whether cePET/MR imaging offers accuracy that is the same as or better than that of cePET/CT in the characterization of PET-positive lesions and whether PET/MR imaging with only T2w fat-suppressed images (T2w PET/MR imaging) might be a justifiable alternative to cePET/CT, as fluid-sensitive sequences in PET/MR imaging might obviate additional MR contrast application.

\section{MATERIALS AND METHODS}

\section{Patient Population}

A total of 150 adult patients (114 men, 36 women; mean age, $64 \mathrm{y}$; range, 27-91 y) were enrolled in this prospective study. All patients were referred for a clinical ${ }^{18} \mathrm{~F}$-FDG PET/CT examination for staging, restaging, or follow-up of various head and neck cancers. Follow-up scans were performed at least 3 mo after surgery or radiation therapy. Exclusion criteria were unwillingness to undergo an additional MR examination, claustrophobia, MR-incompatible medical devices (e.g., cardiac pacemakers, neurostimulators, cochlear implants, and insulin pumps), possible metallic fragments in the body, or renal insufficiency (i.e., glomerular filtration rate $<60 \mathrm{~mL} / \mathrm{min}$ ). This study was approved by the institutional ethics committee, and written informed consent was obtained from all patients before the examination.

\section{PET/CT and MR Imaging}

Sequential PET/CT, ceCT, and ceMR imaging were performed on a trimodality PET/CT-MR setup (full-ring, time-of-flight Discovery PET/CT 690 and 3-tesla Discovery MR750w; both GE Healthcare). The dedicated MR- and CT-compatible shuttle transfer mechanism connecting the MR system and the PET/CT system allowed for PET/CT scanning free of radiofrequency coil-induced artifacts and ascertained the placement of dedicated radiofrequency coils for MR imaging without repositioning of the patient $(13,14)$.

In accordance with the EANM procedure guidelines for PET imaging, patients fasted for at least $4 \mathrm{~h}$ before injection of a standard dose of approximately $330 \mathrm{MBq}$ of ${ }^{18} \mathrm{~F}-\mathrm{FDG}$ (15). After an uptake time of $30 \mathrm{~min}$, the patients were positioned on the shuttle table in the MR suite and the MR acquisition covering the region between the orbital roof and the cranial end of the sternum was started. The images were acquired using a dedicated radiofrequency coil (16-channel highdensity head-neck-spine coil; GE Healthcare). The MR pulse sequences applied included an axial T1w 3-dimensional spoiled gradient echo pulse sequence (LAVA pulse sequence; GE Healthcare), an axial 2-point Dixon-based T2w gradient echo sequence (IDEAL pulse sequence; GE Healthcare), an axial 2-point Dixon-based ceT1w gradient echo sequence (IDEAL pulse sequence; GE Healthcare), coronal and sagittal 2-point Dixon-based ceT1w gradient echo sequences (LAVA Flex; GE Healthcare), and an axial diffusion-weighted imaging sequence. All images were acquired with a slice thickness of $4 \mathrm{~mm}$ within a total MR scan duration of 20-25 min (additional scanning parameters are provided in supplemental material available at http:// jnm.snmjournals.org). The intravenously injected amount of contrast medium (gadodiamide [Omniscan]; GE Healthcare) was $0.2 \mathrm{~mL} / \mathrm{kg}$ of body weight with an injection speed of $1.5 \mathrm{~mL} / \mathrm{s}$. Because the T2 signal properties are not significantly affected by the presence of gadolinium, intravenous contrast medium was injected before the acquisition of the T2w images to allow for a consistent accumulation of gadolinium in the postcontrast sequences.

After shuttle transfer to the adjacent PET/CT system, unenhanced low-dose CT and PET emission data were acquired after a standardized uptake time of $60 \mathrm{~min}$ (range, 60-65 $\mathrm{min}$ ) from the mid thigh to the vertex of the skull. Directly after the acquisition of the PET data, $70 \mathrm{~mL}$ of intravenous contrast medium (iodixanol [Visipaque 320]; GE Healthcare) were injected with a speed of $3 \mathrm{~mL} / \mathrm{s}$. The ceCT scan, with scan coverage in accordance with the MR imaging, was started $60 \mathrm{~s}$ after the beginning of the contrast injection. Low-dose CT and regular-dose ceCT scans were acquired in breath-hold. Scan parameters were as follows: tube voltage, $120-140 \mathrm{kV}$; tube current with automated dose modulation, 60-440 mA/slice; collimation, $64 \times 0.625$; pitch, 0.984:1; rotation time, $0.5 \mathrm{~ms}$; coverage speed, $78 \mathrm{~mm} / \mathrm{s}$; field of view, $50 \mathrm{~cm}$; and images with a transverse pixel size of 0.625 and a slice thickness of $3.75 \mathrm{~mm}$ reconstructed in the axial, coronal, and sagittal planes.

PET data were acquired in 3-dimensional time-of-flight mode with a scan duration of 2 min per bed position, a bed position overlap of $23 \%$, and an axial field of view of $153 \mathrm{~mm}$. The emission data were corrected for attenuation by use of the low-dose CT and were iteratively reconstructed (matrix size, $256 \times 256$; Fourier rebinning [voxel imaging PET mode]; VUE Point FX algorithm [GE Healthcare], 3-dimensional, with 3 iterations and 18 subsets).

The imaging procedure is summarized in Table 1 (13).

\section{Image Processing}

The acquired PET, CT, and MR images were transmitted to a dedicated review workstation (Advantage Workstation, version 4.5; GE Healthcare), which enables review of the PET, CT, and MR images side by side or in fused/overlay mode (PET/CT; PET/MR imaging). Because of the calibrated 3-modality system, no softwarebased image registration was necessary. A previously conducted study validated the image registration accuracy, with less than 4-mm lateral misalignment between CT, PET, and MR datasets, similar to the intrinsic error assessed with phantom measurements (16).

\section{Image Analysis}

All images were analyzed by 2 physicians board-certified in both nuclear medicine and radiology. First, each PET examination was evaluated for the presence of PET-positive lesions within the head and neck area that was covered by the ceCT and the ceMR imaging. Lesions were considered PET-positive if their maximum standardized uptake value (SUV) was at least 2-fold higher than the surrounding 


\begin{tabular}{ll}
\hline Step & Description \\
\hline 1 & Injection of ${ }^{18}$ F-FDG after at least $4 \mathrm{~h}$ of fasting \\
2 & 30 min after injection, transfer of patient onto shuttle board into MR scanner \\
3 & Acquisition of MR sequences $(\sim 25 \mathrm{~min})$ \\
4 & Removal of coils from patient without moving patient (shuttle board slides patient into and out of dedicated \\
& head and neck coil) \\
5 & Transfer of patient on shuttle board to PET/CT scanner ( $<2$ min) \\
7 & Acquisition of standard PET/CT scan with ceCT at end of procedure $(\sim 20 \mathrm{~min})$ \\
\hline
\end{tabular}

background activity. Symmetric physiologic uptake in the lymphatic tissue (e.g., Waldeyer ring), arytenoid muscle uptake, or sinusitis was not included. Lesions were selected in consensus to make sure that both readers evaluated the same lesion. In a second step, each reader analyzed $50 \%$ of the cePET/CT images and $50 \%$ of the cePET/MR images, while masked to the corresponding other modality. Regarding assessment of the PET/MR imaging, first the PET/MR imaging with only axial T2w fat-suppressed images (T2w PET/MR imaging) was analyzed, in a second step the PET/MR imaging with axial ceT1w images (T1w cePET/MR imaging) was analyzed, and in a final step all acquired images (cePET/MR imaging) were considered. At the end, a consensus reading was performed to determine on a case-by-case basis the superiority of CT or MR.

Image quality was assessed on a 3-point scale, with 1 indicating absence of relevant artifacts; 2 , the presence of mild artifacts with sufficient image quality for morphologic assessment; and 3, the presence of substantial artifacts with insufficient image quality for further assessment.

Lesion conspicuity was graded on a 4-point scale based on the percentage of distinct circumferential delineation, that is, $<25 \%$, $25 \%-49 \%, 50 \%-75 \%$, or $>75 \%$. Grade 1 represents lesions with only limited morphologic recognizability, whereas grade 4 implies almost complete lesion delineation.

To determine the impact of the different morphologic images on lesion characterization, each reader had to determinate on a case-bycase basis whether one of the modalities considerably increased the diagnostic confidence or if they performed equally.

Signs of extracapsular spread (ECS) of lymph node metastasis were determined for each evaluated lymph node. Commonly accepted structure alterations implying ECS are irregular margins of the lymph node capsule, capsular enhancement, and infiltration of adjacent fat or muscular tissue.

Quantitatively assessed were the lesion size (measured on the basis of the maximal diameter and perpendicular diameter on the axial images, i.e., the long and the short axis in lymph nodes), maximum SUV, and the ${ }^{18} \mathrm{~F}-\mathrm{FDG}$ PET-based tumor metabolic volume. The tumor metabolic volume was determined by a threshold value of $43 \%$ maximum SUV. In lesions with only faint ${ }^{18}$ F-FDG uptake, a threshold of 3 SDs above background activity was applied.

\section{Statistical Analysis}

All statistical tests were performed using the SPSS Statistics software package for Microsoft Windows (SPSS Version 20; IBM Corp.). $P$ values of less than 0.05 were considered statistically significant. The Wilcoxon signed ranks test was used for comparison of lesion conspicuity and of image artifacts on CT and MR imaging.

\section{RESULTS}

Eighty-five of the 150 patients examined with cePET/CT and cePET/MR imaging (60 men, 25 women; mean age, $67 \mathrm{y}$; range, $27-87$ y) presented with at least one ${ }^{18}$ F-FDG-positive lesion in the head and neck region remaining for further evaluation. The number of ${ }^{18} \mathrm{~F}-\mathrm{FDG}$-positive lesions assessed totalled 149. Among these patients, an additional thirteen ${ }^{18} \mathrm{~F}-\mathrm{FDG}-$-negative lesions with potential clinical impact were detected. Consequently, 162 lesions were evaluated. Thirty percent of the patients were scanned for initial staging, and $70 \%$ of the patients for follow-up. All patients tolerated the procedure well, as they had emptied the bladder before ${ }^{18} \mathrm{~F}-\mathrm{FDG}$ injection and the diuretic load due to MR and CT contrast medium was given 45 min after the start of the procedure. There were no complaints of uncomfortable bladder distention due to osmosis-induced diuresis.

Quantitative PET measurements yielded a median metabolic volume of $4.0 \mathrm{~mL}$ (range, $0.3-53 \mathrm{~mL}$ ) and a mean maximum SUV of 10.2 (range, 2.3-39.9). The ${ }^{18}$ F-FDG-positive lesions were attributed according to histology, clinical inspection, or follow-up to the following entities: 44 primary tumors, 66 lymph nodes, 4 distant metastases, 22 inflammatory lesions, and 13 unspecific accumulations considered physiologic (mainly affecting the tongue).

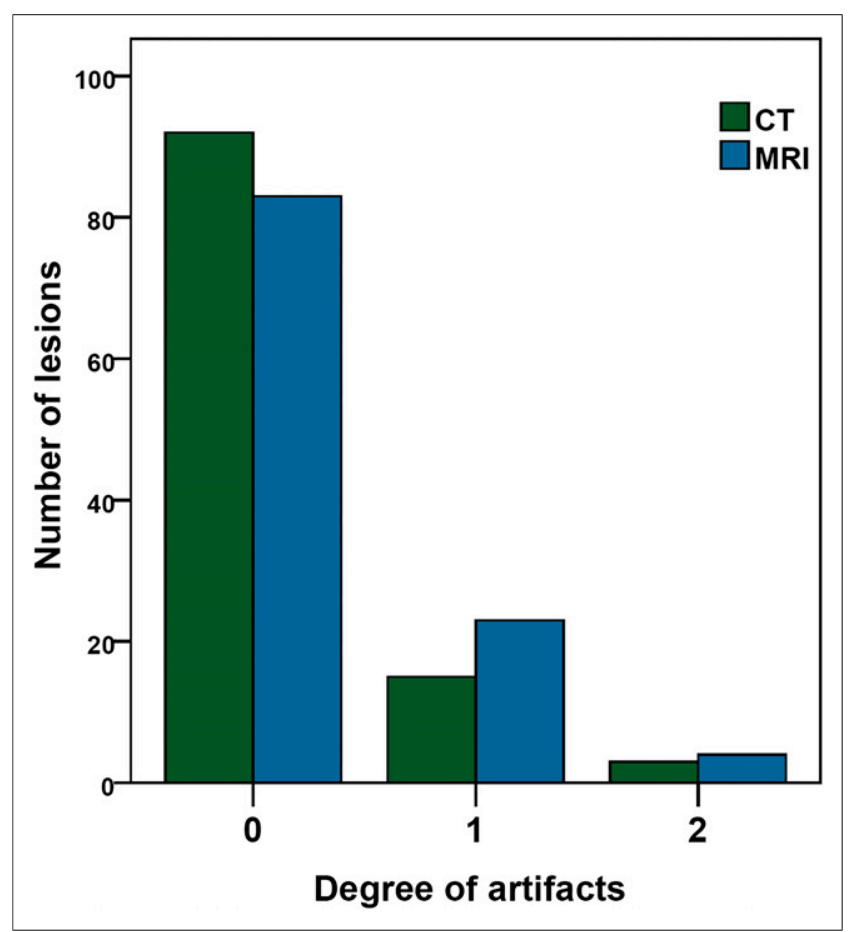

FIGURE 1. Image quality: absence of artifacts (0); mild artifacts (1); substantial artifacts (2). 
The primary tumors were located in the nasopharynx $(n=8$; $18.2 \%)$, sinonasal cavities $(n=3 ; 6.8 \%)$, oropharynx $(n=9$; $20.5 \%)$, oral cavity $(n=6 ; 13.6 \%)$, hypopharynx $(n=5$; $11.4 \%)$, larynx $(n=6 ; 13.8 \%)$, and salivary glands, thyroid, skin, or bone $(n=7 ; 15.9 \%)$. The distributions of metastatic lymph nodes in the cervical levels according to the guidelines of the American Joint Committee on Cancer were 14 in level I (21.2\%), 22 in level II (33.2\%), 11 in level III (16.6\%), 4 in level IV $(6.0 \%), 6$ in level V (9.1\%), 1 in level VI (1.5\%), 2 in level VII (3.0\%), 4 retropharyngeal/Rouvière $(6.0 \%)$, and 2 buccal/parotid (3.0\%). Distant metastases in the head and neck area were detected in bone $(n=2)$ or in soft tissue $(n=2)$. The inflammatory lesions were attributed to osteomyelitis or osteonecrosis $(n=$ $5)$, reactive lymph nodes $(n=4)$, postradiogenic ulcera $(n=2)$, dental root infection $(n=2)$, thyroiditis $(n=2)$, and focal sinusitis, unilateral tonsillitis, carotid plaque, parapharyngeal abscess, proximal esophagitis, or paratracheostomy and sublingual gland inflammation (all $n=1$ ). ${ }^{18} \mathrm{~F}-\mathrm{FDG}$-negative lesions with potential clinical impact were thyroid nodules or cysts $1 \mathrm{~cm}$ or larger in 8 patients $(9.4 \%)$, a necrotic lymph node in 2 patients $(2.4 \%)$, a postoperative chylus fistula in 1 patient, a postoperative seroma in 1 patient, and a small meningioma of the greater wing of the sphenoid bone in 1 patient (1.2\%, respectively). One of the thyroid nodules was not detected by MR imaging because of swallowing/ breathing artifacts, and the meningioma was not detected by the ceCT because of its small size. All other lesions were recognizable in ceCT and MR imaging. Diffusion-weighted imaging as part of the PET/MR imaging protocol did not benefit lesion detection in the presence of ${ }^{18} \mathrm{~F}$-FDG.

\section{Image Quality}

Fewer artifacts reduced image quality in PET/CT (13.6\% grade I and $2.7 \%$ grade II) than in PET/MR imaging (20.9\% grade I and $3.6 \%$ grade II), but no significance level was reached (Wilcoxon signed rank test, $P=0.15$; Fig. 1 ). In CT, most artifacts are found in the suprahyoid region, whereas in MR imaging most artifacts are in the infrahyoid level (Fig. 2).

\section{Lesion Conspicuity}

Regarding the conspicuity of primary tumors (Fig. 3A), T1w cePET/MR imaging and T2w PET/MR imaging performed signifi-

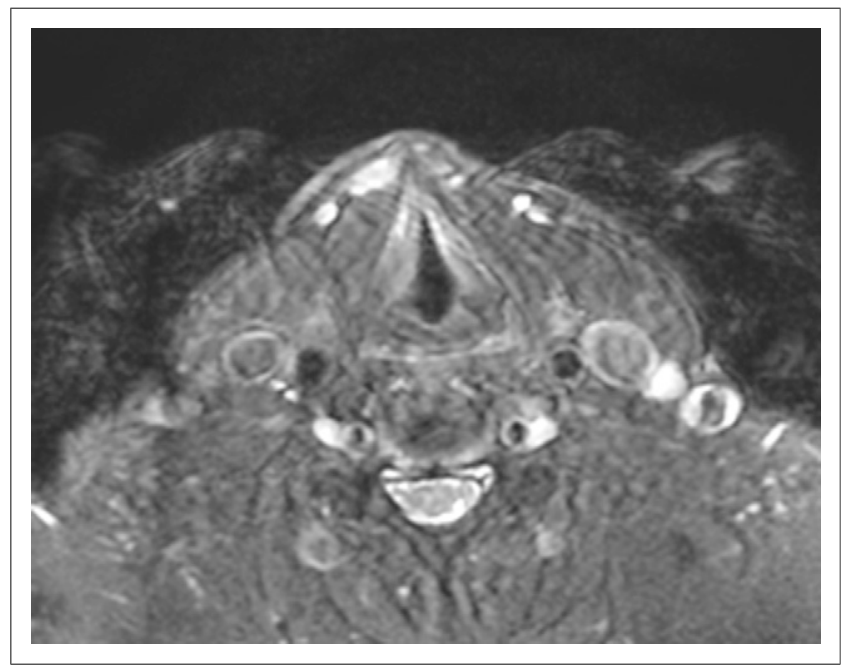

FIGURE 2. Ghosting artifacts along phase-encoding direction due to motion.

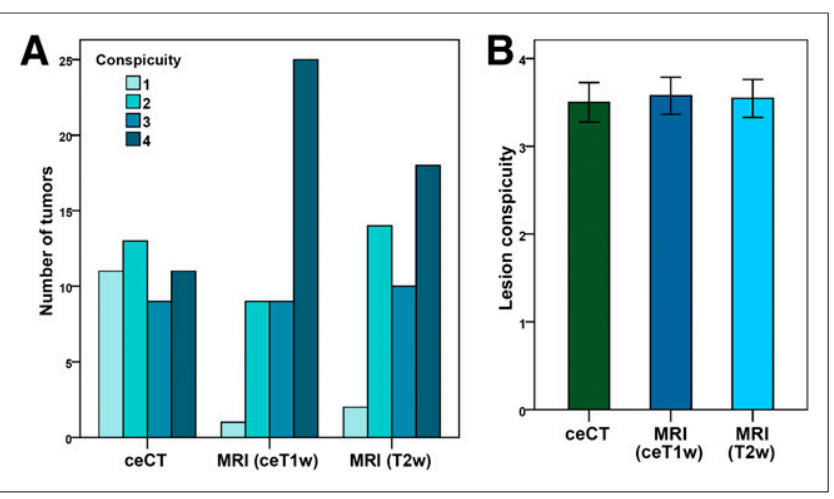

FIGURE 3. Conspicuity of lesion grades $1-4$, that is, $<25 \%, 25 \%-$ $49 \%, 50 \%-75 \%$, or $>75 \%$ circumferential delineation. (A) Conspicuity of primary tumors. (B) Conspicuity of cervical lymph node metastases. Error bars indicate \pm 2 SDs.

cantly better then cePET/CT (Wilcoxon signed rank test, $P=0.001$ and $P=0.002$, respectively). Also, the performance of T1w cePET/ MR imaging was considered superior to T2w PET/MR imaging (Wilcoxon signed rank test, $P=0.007$ ). Regarding the conspicuity of PET-positive lymph nodes (Fig. 3B), no significant difference was found between cePET/CT, T1w cePET/MR imaging, and T2w PET/MR imaging (Wilcoxon signed rank test, $P<0.01$ ).

\section{Diagnostic Confidence}

Regarding tumor characterization, T1w cePET/MR imaging was considered superior to cePET/CT in $50 \%$, equal to cePET/CT in $38.6 \%$, and inferior to cePET/CT in $11.4 \%$. T2w PET/MR imaging was considered superior to cePET/CT in $34.1 \%$, equal to cePET/CT in $50 \%$, and inferior to cePET/CT in $15.9 \%$. Regarding lymph node assessment, no significant difference was found, with $\mathrm{T} 1 \mathrm{w}$ cePET/MR imaging being superior to cePET/CT in $9.1 \%$, equal to cePET/CT in $84.8 \%$, and inferior to cePET/CT in $6.1 \%$. $\mathrm{T} 2 \mathrm{w}$ PET/MR imaging was considered superior to cePET/CT in $7.6 \%$, equal to cePET/CT in $84.8 \%$, and inferior to cePET/CT in $7.6 \%$ (Fig. 4). Signs of ECS were found in $23 \%$ in ceCT/PET, in $23 \%$ in T1w cePET/MR imaging, and in $20 \%$ in T2w PET/MR imaging.

Perineural spread was detected by ceMR imaging in 3 of the 44 primary tumors $(7 \%)$, whereas 1 was also visible on PET. Coronal and sagittal images provided important information in most of the cases in which exact tumor infiltration had to be determined, especially in the nasopharynx (Hiatus of Morgagni/skull base), the tongue (neurovascular bundle), and the larynx (paralaryngeal space). In $9 \%$ of the tumor lesions, potential cartilage or bone infiltration was not conclusive on cePET/MR imaging and CT was helpful in the detection of cortical erosions of the mandibula ( 2 patients) and of the skull base (1 patient). In another patient, infiltration of the laryngeal skeleton was considered unlikely given the appearance of the thyroid cartilage on CT.

Regarding lesions without histologic verification, the follow-up period was between 6 mo and $1 \mathrm{y}$.

\section{DISCUSSION}

The concept of the present study was to characterize ${ }^{18} \mathrm{~F}-\mathrm{FDG}-$ positive lesions in the corresponding morphologic CT and MR images with and without the application of intravenous contrast medium and to understand which portions of the imaging information 


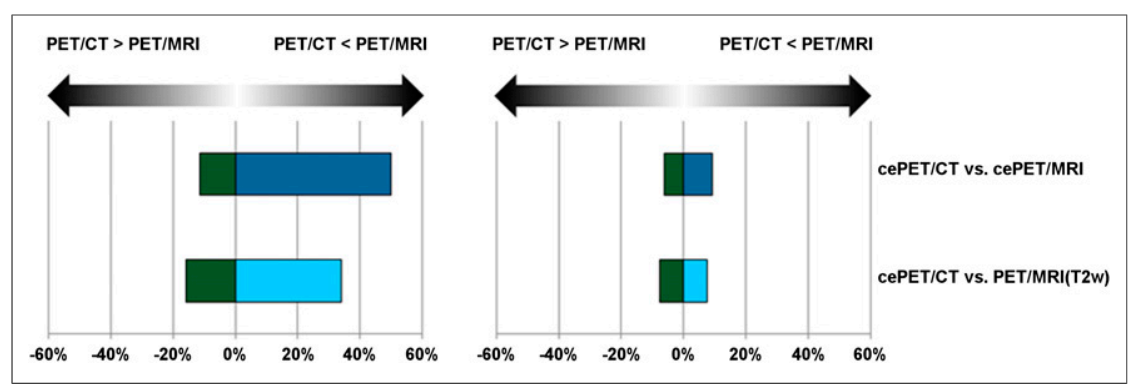

FIGURE 4. Superiority of modalities for assessment of primary tumors (left) and of lymph node metastases (right). Blue implies higher impact of MR imaging, whereas green implies higher impact of CT.

enhance the sensitivity and specificity of the hybrid examination and which portions are redundant.

Because of the rather unspecific accumulation of ${ }^{18} \mathrm{~F}-\mathrm{FDG}$ in neoplastic and inflammatory cells and the limited spatial resolution of PET, coregistered morphologic images are necessary to increase the specificity of the examination and to analyze the relationship of neoplastic tissue to its surroundings. The high softtissue contrast and the different functional imaging techniques of MR imaging might help to ameliorate the informative value of a hybrid imaging study. However, primary tumor staging, therapy response assessment, or early detection of tumor recurrence may not require the same imaging protocols. It has to be considered that ${ }^{18} \mathrm{~F}$-FDG already acts as a contrast agent and under certain circumstances MR contrast may not yield additional clinically relevant information.

The sequential PET/CT and MR imaging was performed on a trimodality PET/CT-MR system that allowed for direct comparison of coregistered PET/CT and PET/MR images. The aim of the present study was to collect clinical imaging data and to contribute to protocol optimization in the restricted time setting of clinical PET/ MR. The presented data do not address the issue of MR-based attenuation correction, but we assume that eventually reliable MR-based attenuation correction will be possible. This prognosis is supported by other studies that showed that PET/MR performs equivalently to PET/CT in terms of qualitative lesion detection $(17,18)$.

For CT and MR imaging, no significant difference in image quality and robustness was found. However, in CT most artifacts are in the suprahyoid region, that is, in the oral cavity and the oropharynx, mainly because of dental implants (Fig. 5), whereas in MR imaging most artifacts are in the infrahyoid region and are mainly due to swallowing or respiration. Most lymph node metastases are in level II and therefore their CT appearance might be affected by CT artifacts, whereas lymph node involvement of the lower neck levels is less common.

Compared with cePET/CT, tumor conspicuity was significantly higher with T1w cePET/MR imaging and with T2w PET/MR imaging. In addition, PET/MR imaging better visualized the midline structures to detect neoplastic midline crossing and also differentiated more reliably between tumor tissue and adjacent or entrapped mucus in the sinonasal cavities. Generally, T1w cePET/MR imaging enabled the most accurate tumor delineation. However, T2w PET/ MR imaging provided similar accuracy in oro- and hypopharyngeal lesions and in sinonasal involvement and may even better delineate the tumor in the oral cavity, particularly in the tongue. T2w PET/ MR imaging performed similarly to cePET/CT in tumor delineation in the nasopharynx and in the larynx; however, both techniques were inferior to T1w cePET/MR imaging. Furthermore T1w cePET/MR imaging considerably better visualized the infiltration of adjacent fascia, muscles, vessels, and cranial nerves; perineural spread; and intracranial complications. Regarding perineural spread, with the higher resolution of the time-of-flight scan technique, PET more often visualizes tumor growth along various cranial nerves too (Fig. 6). MR imaging has been shown to have a higher sensitivity, specificity, and negative predictive value than $\mathrm{CT}$ in the detection of laryngeal cartilage infiltration (19). A high T2 signal compared with normal cartilage and strong contrast enhancement is typical for reactive and inflammatory changes, whereas neoplastic infiltration shows an intermediate T2 signal and moderate contrast enhancement (19).

Considering potential infiltration of bony structures such as the mandibula or the skull base, previous studies comparing MR imaging and CT found a high sensitivity and a high negative predictive value for both modalities (Fig. 7) (20). False-positive findings have been attributed to inflammatory or reactive changes and to radioosteonecrosis (21). High-resolution CT might be able to characterize these lesions with a higher specificity and may also detect subtle cortical erosions. In the present study, in only $5 \%$ of the cases (4/85) did CT add essential information to detect bone or cartilage involvement.

The diagnostic confidence was considered higher with T1w cePET/MR and T2w PET/MR imaging than with PET/CT in 50\% and $34 \%$, respectively. The more reliable determination of potential infiltration of adjacent structures by tumor tissue leads to a higher impact of PET/MR imaging. Especially in the nasopharynx and larynx, T1w cePET/MR images showed superiority over T2w PET/MR images. This is of particular importance because the $T$ classification of nasopharyngeal and laryngeal cancer depends on the infiltration of adjacent structures (22). PET/CT was considered to have a higher impact if respiration or swallowing artifacts deteriorated the MR images or if cortical bone showed morphologic alterations (Table 2).

In accordance with previous studies comparing ceCT and ceMR imaging (23), no significant difference was found between cePET/ $\mathrm{CT}, \mathrm{T} 2 \mathrm{w}$ PET/MR imaging, and cePET/MR imaging in the detection and characterization of cervical lymph node metastases. Previous studies have shown that cePET/CT is advisable in patients with head and neck tumors for the correct diagnosis of necrotic or cystic lymph node metastases (3). Cystic lymph nodes are particularly common in human papillomavirus-positive tonsillar cancer,

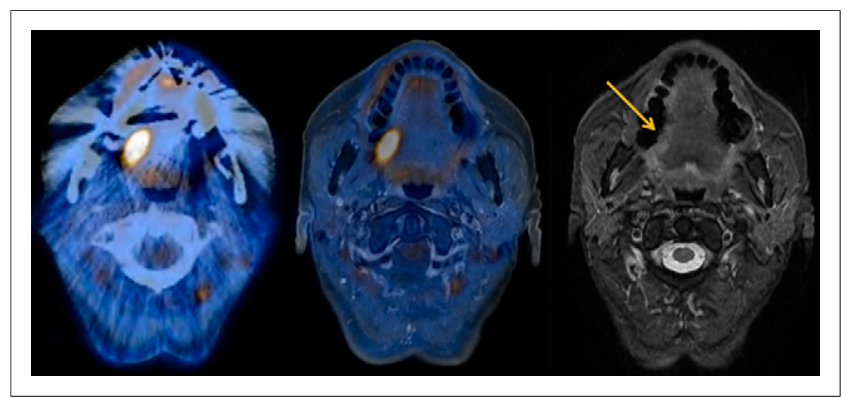

FIGURE 5. Artifacts by dental implants: squamous cell carcinoma of right lateral border of tongue (arrow). From left to right are shown PET/ CT, PET/MR imaging, and T2W MR imaging. 


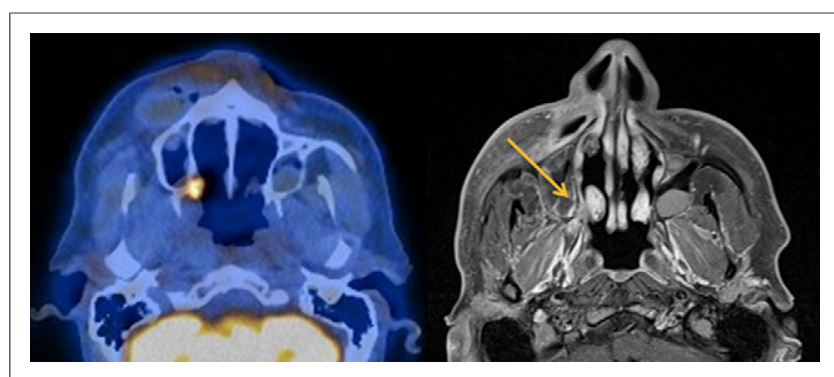

FIGURE 6. Perineural spread of adenoid cystic carcinoma along greater palatine canal on right (arrow), as shown on PET/CT (left) and T1w ceMR imaging (right).

which may manifest as cancer of unknown origin, or in papillary thyroid cancer. The presented results confirm the hypothesis that T2w PET/MR imaging is adequate to detect cystic or necrotic lymph nodes (Fig. 8).

Importantly, to prevent the false interpretation of a fatty hilus as fluid or necrosis, T2w images should be acquired under fat suppression.

In addition to tissue necrosis in lymph nodes, ECS is a further sign of malignancy. Our results confirm the conclusion of previous studies that MR imaging is as reliable as ceCT for detecting ECS (24). T2w $\mathrm{PET} / \mathrm{MR}$ imaging was insignificantly inferior to cePET/MR imaging in the detection of ECS. A benefit of ceT1w images might be the better determination of potential infiltration of adjacent structures such as vessels, muscles, and fasciae. Mentionable is a PET/CT study by Kubicek at al. that showed SUV measured in a lymph node to be predictive for ECS and for distant recurrence (25).

The incidence of distant metastases in head and neck cancer is relatively small in comparison to other malignancies. The most common locations for distant metastases of squamous cell cancer are lung (66\%), bone (22\%), and liver (10\%) (26). Especially, lung imaging is considered a challenge for MR imaging. However, it has already been shown using a 3-dimensional Dixon-based, dualecho gradient recalled echo pulse sequence that although the detection rates of pulmonary nodules were lower, there was no statistically significant difference in a patient-based evaluation compared with low-dose CT (27). Considering the limited data on PET/MR lung imaging, it might be advisable to perform CT of the lung if the MR lung scan is negative in a patient with a high likelihood of lung metastasis, until PET/MR lung imaging is validated by further studies.

Because of the focus on sequence selection in head and neck PET/MR imaging, the data presented in this study make no statement with regard to the capabilities of whole-body MR to detect distant metastasis. In the present study, 4 distant metastases were found in the head and neck region covered by cePET/CT and by cePET/MR imaging. All metastases were detected with both modalities and were concordantly attributed to bone or soft tissue.

The challenge in ${ }^{18} \mathrm{~F}$-FDG PET is the unspecific accumulation of tracer in tumor tissue and inflammatory cells. In MR imaging, neoplastic tissue and inflammatory reactions might be differentiated on the basis of their signal intensities and contrast accumulation. Inflammatory lesions generally have a high signal on $\mathrm{T} 2 \mathrm{w}$ and ceT1w images, whereas tumor tissue has an intermediate T2 signal and only moderate contrast enhancement. However, this quantification of signal intensity is difficult to standardize, and for the correct attribution of an ${ }^{18} \mathrm{~F}-\mathrm{FDG}$ accumulation to tumor or inflammation, other parameters have to be taken into consider- ation. Therefore, this potential benefit of MR requires further evaluation.

It has been found that, despite the 3-dimensional information from PET, morphologic CT or MR images in the coronal and sagittal plane are of major importance for high diagnostic confidence and for the planning of surgery or radiation therapy. Coronal planes are particularly helpful in assessment of the paralaryngeal space, the neurovascular bundle of the tongue, and the anterior skull base. Sagittal planes are notably supportive for the assessment of the preepiglottic fat space and the posterior skull base.

However, in view of the lengthy time required for MR data acquisition, it might be legitimate to cover only the volume from the tip of the tongue to the prevertebral space in the coronal plane and the pharynx and parapharyngeal space in the sagittal plane. Because the most critical structures are imaged with this coverage, a major reduction in acquisition time may be achieved.

Especially in the restricted time setting of clinical PET/MR, only images with a potential impact on therapy management should be included in a protocol. T2w PET/MR imaging has a diagnostic performance similar to (metastatic lymph nodes) or higher than (primary tumors) that of cePET/CT in the morphologic characterization of PET-positive lesions. However, on the basis of our findings it seems justified, despite the ${ }^{18} \mathrm{~F}-\mathrm{FDG}$ "contrast-agent," to recommend the application of MR contrast medium and the acquisition of axial, coronal, and sagittal planes in primary staging when infiltration of adjacent structures is an important issue. Also, for the detection of potential perineural spread, cePET/MR imaging provides the highest sensitivity.

In therapy response assessment, a reduced MR protocol might be considered, because PET is the major component for the distinction of responders from nonresponders. The additional scanning time may be used for other promising and - concerning lesion characterizationmore sophisticated MR techniques such as diffusion-weighted imaging, intravoxel incoherent motion, or arterial spin labeling to increase the specificity of the hybrid imaging examination. Finally, $\mathrm{T} 2 \mathrm{w}$ PET/MR imaging might act as a survey sequence if the prob-

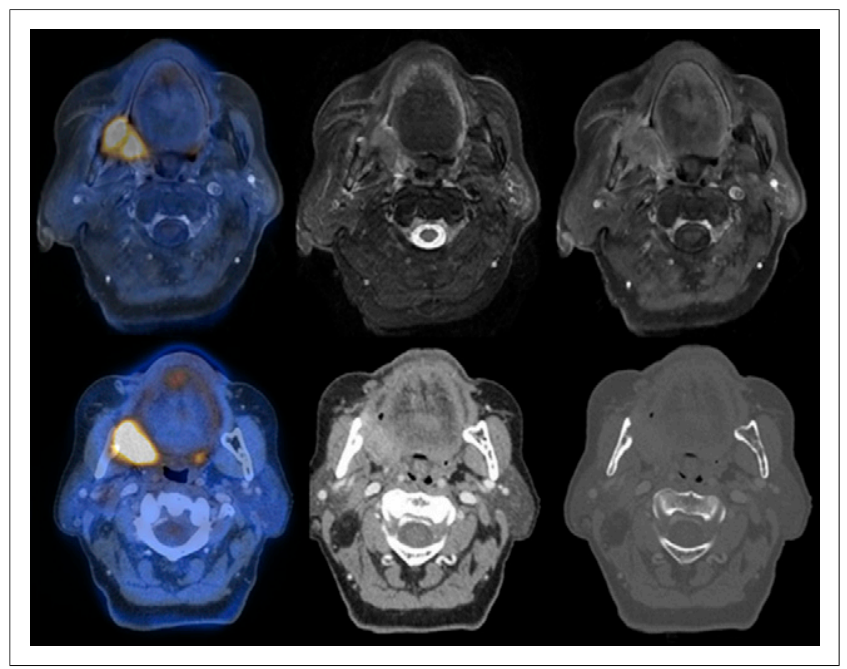

FIGURE 7. Mandibular invasion of squamous cell carcinoma of right retromandibular trigon. (Top row, from left to right) PET/MR imaging, T2w MR imaging, and T1w ceMR imaging. (Bottom row, from left to right) $\mathrm{PET} / \mathrm{CT}$, ceCT, and high-resolution $\mathrm{CT}$ with window size and level optimized for bone assessment. 
TABLE 2

Mean Lesion Conspicuity in Different Compartments

\begin{tabular}{|c|c|c|c|c|c|}
\hline \multirow[b]{2}{*}{ Site } & \multicolumn{3}{|c|}{ Mean lesion conspicuity* } & \multicolumn{2}{|c|}{ Diagnostic confidence $^{\dagger}$} \\
\hline & cePET/CT & cePET/MR imaging & T2w cePET/MR imaging & cePET/MR imaging & T2w cePET/MR imaging \\
\hline Nasopharynx & 2.3 & 3.1 & 2.4 & 0.9 & 0 \\
\hline Sinonasal cavities & 4.0 & 4.0 & 3.7 & 0 & -0.3 \\
\hline Oropharynx & 1.9 & 3.3 & 3.1 & 0.6 & 0.6 \\
\hline Oral cavity & 2.3 & 3.3 & 3.0 & 0.3 & 0.3 \\
\hline Hypopharynx & 2.0 & 3.0 & 2.6 & 0.6 & 0.6 \\
\hline Larynx & 2.3 & 3.0 & 2.8 & 0.3 & 0 \\
\hline
\end{tabular}

*Grades 1-4, with 4 representing highest degree of morphologic recognizability.

†'Superiority of modalities regarding diagnostic confidence, as graded with numeric values between -1 and 1 . Grade of -1 implies higher diagnostic impact of PET/CT, whereas grade of 1 implies higher diagnostic impact of PET/MR imaging.

ability of head and neck cancer is low, that is, in a whole-body protocol for another tumor entity or in follow-up settings without any clinical suspicion of tumor recurrence. The $\mathrm{T} 2 \mathrm{w}$ sequence together with the PET information has a high negative predictive value because necrotic lymph nodes are also detected whereas PET alone may fail to show a pathologic accumulation.

In accordance with another study by Buchbender et al. (28), diffusion-weighted imaging as part of the PET/MR imaging protocol did not benefit lesion detection. However, a potential impact of a combined diffusion-weighted imaging-PET/MR imaging protocol on therapy response analysis was not assessed.

An additional CT scan (on a stand-alone CT device) without contrast medium might be indicated in rare cases after the PET/ MR examination if potential bone or cartilage infiltration is doubtful on MR imaging, if artifacts have deteriorated image quality, or if PET/MR imaging findings are negative in a patient with a high likelihood of lung metastasis.

In agreement with the prospective study design and the assessment of 150 consecutive patients, no particular tumor entity was preferentially analyzed. Therefore, the number of tumors with potential infiltration of critical structures such as the skull base, the mandibula, or the laryngeal cartilage is limited and further studies with particular selection of these entities are necessary.

\section{CONCLUSION}

The results of the present study provide evidence that PET/MR imaging is a legitimate alternative to PET/CT in the clinical workup of patients with head and neck cancers. Intravenous MR contrast medium may be applied only if the exact tumor extent or infiltration of crucial structures is of concern (i.e., preoperatively) or if perineural spread is anticipated. In early assessment of response to therapy, in follow-up examinations, or in a whole-

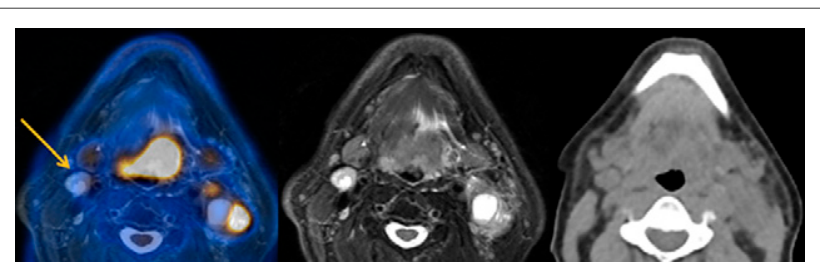

FIGURE 8. Necrotic lymph node with missing ${ }^{18} \mathrm{~F}-\mathrm{FDG}$ accumulation (arrow). From left to right are shown T2w PET/MR imaging, T2w MR imaging, and CT without contrast medium. body protocol for non-head and neck tumors, T2w PET/MR imaging might be sufficient for coverage of the head and neck part. The additional MR scanning time may rather be used for advanced MR techniques to increase the specificity of the hybrid imaging examination. An additional CT scan without contrast medium might be indicated in rare cases if PET/MR imaging is not fully conclusive.

\section{DISCLOSURE}

The costs of publication of this article were defrayed in part by the payment of page charges. Therefore, and solely to indicate this fact, this article is hereby marked "advertisement" in accordance with 18 USC section 1734 . This research project was supported by an institutional research grant from GE Healthcare. No other potential conflict of interest relevant to this article was reported.

\section{ACKNOWLEDGMENTS}

We thank our technicians for their excellent technical support and Gaspar Delso for his continuing technical advice.

\section{REFERENCES}

1. Veit-Haibach P, Luczak C, Wanke I, et al. TNM staging with FDG-PET/CT in patients with primary head and neck cancer. Eur J Nucl Med Mol Imaging. 2007;34:1953-1962.

2. Gupta T, Master Z, Kannan S, et al. Diagnostic performance of post-treatment FDG PET or FDG PET/CT imaging in head and neck cancer: a systematic review and meta-analysis. Eur J Nucl Med Mol Imaging. 2011;38:2083-2095.

3. Haerle SK, Strobel K, Ahmad N, Soltermann A, Schmid DT, Stoeckli SJ. Contrast-enhanced ${ }^{18} \mathrm{~F}-\mathrm{FDG}-\mathrm{PET} / \mathrm{CT}$ for the assessment of necrotic lymph node metastases. Head Neck. 2011;33:324-329.

4. Hany TF, Steinert HC, Goerres GW, Buck A, von Schulthess GK. PET diagnostic accuracy: improvement with in-line PET-CT system: initial results. Radiology. 2002;225:575-581.

5. Ahmad A, Branstetter BF IV. CT versus MR: still a tough decision. Otolaryngol Clin North Am. 2008;41:1-22.

6. Kim SY, Kim JS, Yi JS, et al. Evaluation of ${ }^{18} \mathrm{~F}-\mathrm{FDG}$ PET/CT and CT/MRI with histopathologic correlation in patients undergoing salvage surgery for head and neck squamous cell carcinoma. Ann Surg Oncol. 2011;18:2579-2584.

7. Gore JC, Manning HC, Quarles CC, Waddell KW, Yankeelov TE. Magnetic resonance in the era of molecular imaging of cancer. Magn Reson Imaging. 2011;29:587-600.

8. Dhermain FG, Hau P, Lanfermann H, Jacobs AH, van den Bent MJ. Advanced MRI and PET imaging for assessment of treatment response in patients with gliomas. Lancet Neurol. 2010;9:906-920.

9. de Bondt RB, Hoeberigs MC, Nelemans PJ, et al. Diagnostic accuracy and additional value of diffusion-weighted imaging for discrimination of malignant 
cervical lymph nodes in head and neck squamous cell carcinoma. Neuroradiology. 2009;51:183-192.

10. Yankeelov TE, Gore JC. Dynamic contrast enhanced magnetic resonance imaging in oncology: theory, data acquisition, analysis, and examples. Curr Med Imaging Rev. 2009;3:91-107.

11. Hauser T, Essig M, Jensen A, et al. Characterization and therapy monitoring of head and neck carcinomas using diffusion-imaging-based intravoxel incoherent motion parameters: preliminary results. Neuroradiology. 2013;55:527-536.

12. von Schulthess GK, Burger C. Integrating imaging modalities: what makes sense from a workflow perspective? Eur J Nucl Med Mol Imaging. 2010;37:980-990.

13. Veit-Haibach P, Kuhn FP, Wiesinger F, Delso G, von Schulthess G. PET-MR imaging using a tri-modality PET/CT-MR system with a dedicated shuttle in clinical routine. MAGMA. 2013;26:25-35.

14. Kuhn FP, Crook DW, Mader CE, Appenzeller P, von Schulthess GK, Schmid DT. Discrimination and anatomical mapping of PET-positive lesions: comparison of CT attenuation-corrected PET images with coregistered MR and CT images in the abdomen. Eur J Nucl Med Mol Imaging. 2013;40:44-51.

15. Boellaard R, O'Doherty MJ, Weber WA, et al. FDG PET and PET/CT: EANM procedure guidelines for tumour PET imaging: version 1.0. Eur J Nucl Med Mol Imaging. 2010;37:181-200.

16. Kuhn FP, Wiesinger F, Wollenweber SD, Samarin A, Von Schulthess G, Schmid D. Sequential integrated PET/CT-MR system: comparison of image registration accuracy of PET/CT versus PET/MR. Presented at: 20th Annual Meeting of the International Society for Magnetic Resonance in Medicine; Melbourne, Australia; 2012.

17. Wiesmüller M, Quick HH, Navalpakkam B, et al. Comparison of lesion detection and quantitation of tracer uptake between PET from a simultaneously acquiring whole-body PET/MR hybrid scanner and PET from PET/CT. Eur J Nucl Med Mol Imaging. 2013;40:12-21.
18. Eiber M, Martinez-Moller A, Souvatzoglou M, et al. Value of a Dixon-based MR/PET attenuation correction sequence for the localization and evaluation of PET-positive lesions. Eur J Nucl Med Mol Imaging. 2011;38:1691-1701.

19. Becker M, Zbaren P, Casselman JW, Kohler R, Dulguerov P, Becker CD. Neoplastic invasion of laryngeal cartilage: reassessment of criteria for diagnosis at MR imaging. Radiology. 2008;249:551-559.

20. van den Brekel MW, Runne RW, Smeele LE, Tiwari RM, Snow GB, Castelijns JA. Assessment of tumour invasion into the mandible: the value of different imaging techniques. Eur Radiol. 1998;8:1552-1557.

21. Yousem DM, Gad K, Tufano RP. Resectability issues with head and neck cancer. AJNR. 2006;27:2024-2036.

22. Edge SB, Byrd DR, Compton CC, Fritz AG, Greene FL, Trotti A. AJCC Cancer Staging Manual. 7th ed. New York, NY: Springer; 2010.

23. Castelijns JA, van den Brekel MW. Imaging of lymphadenopathy in the neck. Eur Radiol. 2002;12:727-738.

24. King AD, Tse GM, Yuen EH, et al. Comparison of CT and MR imaging for the detection of extranodal neoplastic spread in metastatic neck nodes. Eur J Radiol. 2004;52:264-270.

25. Kubicek GJ, Champ C, Fogh S, et al. FDG-PET staging and importance of lymph node SUV in head and neck cancer. Head Neck Oncol. 2010;2:19.

26. Ferlito A, Shaha AR, Silver CE, Rinaldo A, Mondin V. Incidence and sites of distant metastases from head and neck cancer. ORL J Otorhinolaryngol Relat Spec. 2001;63:202-207.

27. Stolzmann P, Veit-Haibach $P$, Chuck N, et al. Detection rate, location, and size of pulmonary nodules in trimodality PET/CT-MR: comparison of low-dose CT and Dixon-based MR imaging. Invest Radiol. 2013;48:241-246.

28. Buchbender C, Hartung-Knemeyer V, Beiderwellen K, et al. Diffusion-weighted imaging as part of hybrid PET/MRI protocols for whole-body cancer staging: does it benefit lesion detection? Eur J Radiol. 2013;82:877-882. 